\title{
Synchronous gastric cancer and carcinoma in situ of the pancreas detected by SPACE
}

\author{
Masashi Inoue ${ }^{1}$, Keishi Hakoda ${ }^{1}$, Hiroyuki Sawada ${ }^{1}$, Ryuichiro Hotta ${ }^{1}$, Ichiro Omori ${ }^{1}$, \\ Kazuaki Miyamoto ${ }^{1}$, Kazuhiro Toyota ${ }^{1}$, Seiji Sadamoto ${ }^{1}$, and Tadateru Takahashi ${ }^{1}$ \\ ${ }^{1}$ Higashihiroshima Medical Center
}

May 31, 2021

\begin{abstract}
Gastric cancer was diagnosed with dilation of the main pancreatic duct. SPACE revealed adenocarcinoma, total gastrectomy with distal pancreatectomy were underwent. More case reports are needed to determine whether adjuvant chemotherapy is indicated for patients with PCIS and whether the extent of lymph node dissection can be safely reduced.
\end{abstract}

\section{Synchronous gastric cancer and carcinoma in situ of the pancreas detected by SPACE}

Masashi Inoue, ${ }^{1}$ Keishi Hakoda, ${ }^{1}$ Hiroyuki Sawada, ${ }^{1}$ Ryuichi Hotta,,${ }^{1}$ Ichiro Ohmori, ${ }^{1}$ Kazuaki Miyamoto, ${ }^{1}$ Kazuhiro Toyota, ${ }^{1}$ Seiji Sadamoto, ${ }^{1}$ Tadateru Takahashi ${ }^{1,2}$

${ }^{1}$ Department of Surgery, National Hospital Organization Higashihiroshima Medical Center, Higashihiroshima, 739-0041, Japan

${ }^{2}$ Department of Gastrointestinal and Transplant Surgery, Applied Life Sciences, Institute of Biomedical and Health Sciences, Hiroshima University, Hiroshima, Japan

\section{Corresponding author:}

Masashi Inoue, MD

Department of Surgery, National Hospital Organization

Higashihiroshima Medical Center

513 Jike, Saijo-cho, Higashihiroshima, Hiroshima, 739-0041, Japan

Tel: +81-824-22-2176; FAX: +81-824-22-4675

Email: inoue.masashi.uv@mail.hosp.go.jp

\section{Abstract}

Gastric cancer was diagnosed with dilation of the main pancreatic duct. SPACE revealed adenocarcinoma, total gastrectomy with distal pancreatectomy were underwent. More case reports are needed to determine whether adjuvant chemotherapy is indicated for patients with PCIS and whether the extent of lymph node dissection can be safely reduced.

\section{Introduction}

The prognosis for patients with pancreatic cancer could be improved if clinicians were able to detect and treat the disease in its early stages $(1,2)$. Using a recent analysis of the Japan Pancreatic Cancer Registry, 
the Japan Pancreas Society reports that the 5-year survival rate in patients with lesions less than 10 mm in diameter is $80.4 \%$, and that it is $85.8 \%$ in patients with stage 0 pancreatic cancer, comprising high-grade pancreatic intraepithelial neoplasia (PanIN-3) and pancreatic carcinoma in situ (PCIS) (3). However, only $0.6 \%$ of patients with pancreatic cancer are diagnosed at stage 0 because of the difficulty of detecting cancer at this early stage (4). We describe herein a patient with PCIS synchronous with early gastric cancer, detected preoperatively using serial pancreatic juice aspiration cytological examination (SPACE).

\section{Case Report}

A 78-year-old man was hospitalized with upper abdominal pain. Gastrointestinal endoscopy showed a 0-IIc lesion on the lesser curvature of the stomach, at the angulus; biopsy revealed signet ring cell carcinoma. Abdominal computed tomography (CT) and magnetic resonance cholangiopancreatography (MRCP) showed that the main pancreatic duct in the body and tail of the pancreas was dilated, but neither scan revealed a lesion consistent with a tumor (Fig 1, Fig 2). Endoscopic ultrasonography (EUS) and intraductal ultrasonography revealed stenosis of the main pancreatic duct with dilation of the caudal pancreatic duct in the body of the pancreas, but they also did not show any mass (Fig 3). The results of endoscopic retrograde cholangiopancreatography (ERCP) with pancreatic juice cytology favored malignancy but did not reveal adenocarcinoma. We performed SPACE by placing a 4-Fr atype nasopancreatic tube (Gadelius Medical, Tokyo, Japan). Three of the 6 cytologic specimens revealed adenocarcinoma (Fig 4). The preoperative diagnosis was stage IA gastric cancer (cT1bN0M0) with stage 0 cancer of the pancreatic body (TisN0M0).

We performed total gastrectomy and distal pancreatectomy with splenectomy. Histopathologic examination found PanIN-3 with lymphocyte infiltration throughout the pancreas, including the tail, with dilation of the caudal pancreatic duct. The postoperative diagnosis was stage 0 pancreatic body cancer (TisN0M0) and stage IA gastric cancer (T1bN0M0) (Fig 5).

The patient required enteral nutrition via a feeding tube for malnutrition after surgery. Two months after surgery, he was discharged in good general condition, although his history of malnutrition precluded the use of adjuvant chemotherapy. There was no evidence of recurrence at his most recent follow-up appointment, 2 years after surgery.

\section{Discussion}

The Japanese guideline for pancreatic cancer recommends ERCP if there are findings suggestive of pancreatic cancer, such as pancreatic duct dilation or stenosis, even if imaging does not show any evidence of a mass (5). Although no pathologic diagnosis was obtained in our patient using pancreatic juice cytology obtained during ERCP, we were able to make the diagnosis using specimens obtained from SPACE. Iiboshi et al compared patients diagnosed using single pancreatic juice cytology versus SPACE, and report that the sensitivity, specificity, and accuracy of SPACE are 100\%, 83.3\%, and 95\%, respectively (6). The advantage of SPACE lies in its better sensitivity for micropancreatic cancer (4).

According to previous reports, patients with early pancreatic cancer are rarely symptomatic but frequently demonstrate focal branch-duct dilation, focal irregular stenosis, small cystic lesions around the area of ductal stenosis, and distal dilation of the main pancreatic duct on EUS and MRCP (4). In patients with PCIS, assessment with ERCP frequently reveals irregularity, noncontinuous narrowing, granular defects, and dilation. Kikuyama et al report that patients with stage 0 disease demonstrate a high degree of fatty change on enhanced CT, in the pancreatic parenchyma around the area of PCIS (7). In certain patients with PCIS, focal pancreatitis with inflammatory cells, desmoplastic changes, fibrosis, and fatty changes are observed in the parenchyma around the area of PCIS (8-10). Ikeda et al classify PCIS into 3 types: flat, low papillary, and mixed (11). Based on this classification system, our patient's disease would be classified as the low papillary type, which may tend to spread intraductally. As PCIS with intraductal spread into the main pancreatic duct and the branch duct can cause focal pancreatitis, patients may experience stenosis and dilation of the main pancreatic duct. We confirmed the presence of lymphocyte infiltration in our patient, and we believe that his stenosis was triggered by inflammation. 
In Japan, the use of an oral fluoropyrimidine (S-1) is indicated for postoperative adjuvant chemotherapy in patients with pancreatic cancer (12). However, there is no evidence of the need for adjuvant chemotherapy for PCIS. More case reports are needed to determine whether adjuvant chemotherapy is indeed indicated for patients with PCIS.

As in our case, patients with both gastric cancer and pancreatic body cancer may require total gastrectomy with distal pancreatectomy, since distal gastrectomy with distal pancreatectomy may leave the patient with venous stasis and insufficient arterial perfusion to the remnant stomach. On the other hand, spleen preserving distal pancreatectomy with conservation of the splenic artery and vein for a pancreatic benign or low grade malignant tumor has accepted (13). Based on a review of the PubMed database, no case of synchronous gastric cancer and PCIS. More case reports are needed to determine whether the extent of lymph node dissection can be safely reduced for advantage of nutrition.

\section{Conclusion}

Because PCIS frequently presents as pancreatic duct stenosis, SPACE is useful for diagnosis.

\section{References}

1. Warshaw AL, del Castillo CF. Pancreatic carcinoma. N Engl J Med. 1992; 326:455-465.

2. Rosewicz S, Wiedenmann B. Pancreatic carcinoma. Lancet 1997; 349:485-489.

3. Egawa S, Toma H, Ohigashi H, et al. Japan Pancreatic Cancer Registry; 30th year anniversary: Japan Pancreas Society. Pancreas 2012; 41:985-992.

4. Kanno A, Masamune A, Hanada K, et al; Japan Study Group on the Early Detection of Pancreatic Cancer (JEDPAC). Multicenter study of early pancreatic cancer in Japan. Pancreatology 2018; 18:61-67.

5. Yamaguchi K, Okusaka T, Shimizu K, et al; Committee for Revision of Clinical Guidelines for Pancreatic Cancer of the Japan Pancreas Society. Clinical practice guidelines for pancreatic cancer 2016 from the Japan Pancreas Society: a synopsis. Pancreas 2017; 46:595-604.

6. Iiboshi T, Hanada K, Fukuda T, et al. Value of cytodiagnosis using endoscopic nasopancreatic drainage for early diagnosis of pancreatic cancer: establishing a new method for the early detection of pancreatic carcinoma in situ. Pancreas 2012; 41:523-529.

7. Kikuyama M, Hanada K, Ueki T. Pancreatic carcinoma in situ presenting prominent fatty change of the pancreatic body on CT: experiences from 3 cases. In Japanese with English Abstract. Suizo 2015; 30:626-632.

8. Miyata T, Takenaka M, Omoto S, et al. A case of pancreatic carcinoma in situ diagnosed by repeated pancreatic juice cytology. Oncology 2017; 93:98-101.

9. Hanada K, Okazaki A, Hirano N, et al. Diagnostic strategies for early pancreatic cancer. J. Gastroenterol $2015 ; 50: 147-154$.

10. Seki M, Ninomiya E, Takano K, et al. Pancreatogram findings for carcinoma in situ (CIS) of the pancreas seen on endoscopic retrograde cholangiopancreatography and postoperative pancreatography of resected specimens: can CIS be diagnosed preoperatively? Pancreatology 2008; 8:142-152.

11. Ikeda M, Yanagisawa A, Seki M, et al. The early state of invasive pancreatic ductal adenocarcinomas: characteristics of the low papillary type and flat type intraductal carcinoma. Pancreas 2006; 33:135-141.

12. Uesaka K, Boku N, Fukutom A, et al.: JASPAC 01 Study Group. Adjuvant chemotherapy of S-1 versus gemcitabine for resected pancreatic cancer: a phase 3, open-label, randomised, non-inferiority trial (JASPAC 01). Lancet $2016 ; 388: 248-257$. 
13. Sugita H, Kuroki H, Akiyama T, et al. Hand-assisted laparoscopic spleen-preserving distal pancreatectomy combined with laparoscopic distal gastrectomy for the treatment of pancreatic neuroendocrine tumor with early gastric cancer: Report of a case. Int J Surg Case Rep. 2016; 28: 258-261.

\section{Figure Legends}

Fig 1: Computed tomography. The main pancreatic duct in the body and tail of the pancreas is dilated, but there is no visible tumor

Fig 2: Magnetic resonance imaging. The main pancreatic duct in the body and tail of the pancreas is dilated, but there is no visible tumor

Fig 3: Endoscopic ultrasonography (a) and intraductal ultrasonography (b). The main pancreatic duct in the body and tail of the pancreas is dilated, but there is no visible tumor

Fig 4: The results of pancreatic juice cytology favor malignancy but do not reveal adenocarcinoma

Fig 5: Postoperative pathologic examination. Pancreatic intraepithelial neoplasia grade 3 is seen in specimens 1 through 4 , and the caudal pancreatic duct is dilated

\section{Consent}

Written informed consent was obtained from the patient for publication of this Case Report and any accompanying images. A copy of the written consent is available for review by the Editor-in-Chief of this journal.

\section{Competing interests}

There are no potential conflicts of interest to disclose.

\section{Author contributions}

M.I. wrote the manuscript and designed the study. R.K., K.H., H.S., R.H., I.O., K.M., K.T., S.S., T.T. proofread the manuscript.

\section{Hosted file}

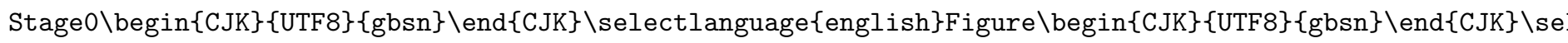
available at https://authorea.com/users/332527/articles/524302-synchronous-gastric-cancerand-carcinoma-in-situ-of-the-pancreas-detected-by-space

\section{Hosted file}

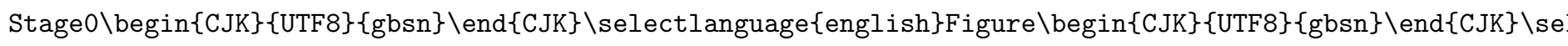
available at https://authorea.com/users/332527/articles/524302-synchronous-gastric-cancerand-carcinoma-in-situ-of-the-pancreas-detected-by-space

\section{Hosted file}

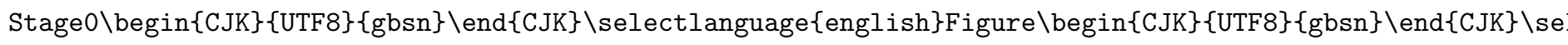
available at https://authorea.com/users/332527/articles/524302-synchronous-gastric-cancerand-carcinoma-in-situ-of-the-pancreas-detected-by-space

\section{Hosted file}

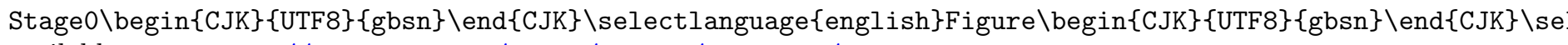
available at https://authorea.com/users/332527/articles/524302-synchronous-gastric-cancerand-carcinoma-in-situ-of-the-pancreas-detected-by-space

\section{Hosted file}


Stage $0 \backslash$ begin $\{\mathrm{CJK}\}\{\mathrm{UTF} 8\}\{$ gbsn $\} \backslash$ end $\{\mathrm{CJK}\} \backslash$ selectlanguage $\{$ english $\} F$ igure $\backslash$ begin $\{\mathrm{CJK}\}\{\mathrm{UTF} 8\}\{$ gbsn $\} \backslash$ end $\{\mathrm{CJK}\} \backslash$ se available at https://authorea.com/users/332527/articles/524302-synchronous-gastric-cancerand-carcinoma-in-situ-of-the-pancreas-detected-by-space 